\title{
Therapeutic Role of Curcumin on Glycemic Regulation, Lipid Parameters and Potential Benefits on Hyperandrogenemia - A Short Communication
}

\author{
Kulvinder Kochar Kaur ${ }^{1 *}$, Gautam Allahbadia ${ }^{2}$ and Mandeep Singh ${ }^{3}$ \\ ${ }^{1}$ Scientific Director, Dr Kulvinder Kaur Centre for Human Reproduction, Jalandhar, \\ Punjab, India \\ ${ }^{2}$ Scientific Director, Ex-Rotunda-A Centre for Human Reproduction, Bandra, \\ Mumbai, India \\ ${ }^{3}$ Consultant Neurologist, Swami Satyanand Hospital, Jalandhar, Punjab, India \\ *Corresponding Author: Kulvinder Kochar Kaur, Scientific Director, Dr Kulvinder \\ Kaur Centre for Human Reproduction, Jalandhar, Punjab, India.
}

Received: December 28, 2021

Published: January 31, 2022

(C) All rights are reserved by Kulvinder

Kochar Kaur., et al.
Polycystic ovary syndrome (PCOS) represents one of most common endocrine condition in case of reproductive women, that mostly presents in the form of a menstrual disorder, hirsutism, infertility [1]. Despite lot of therapies exist like utilization of Oral contraceptives to hamper the maturation of ovarian follicles in the form of long time PCOS treatment, besides ovulation induction for those PCOS patients needing fertility, they can't cure it. Till now lifestyle modifications (like weight reduction) continues to be the 1st line, besides the mainstay of treatment [2]. Earlier we reviewed how to classify in addition to treat PCOS presents especially those with obesity [3]. Furthermore, we discussed the role of therapy with probiotics as one of the innovative treatments in view of no specific therapy working [4].

Recently Chien., et al. [5], conducted a systematic review with meta-analysis and trial sequential analysis (TSA) on the effects of curcumin supplementation in PCOS patients. The major observation of this study was that patients in whom of PCOS where consumption of curcumin occurred displayed significantly higher escalation of glycemic regulation in contrast to those in receipt of placebo that was pointed by fasting glucose, fasting insulin, HOMA- insulin resistance (IR) as well as QUICK1 (Quantitative insulin sensitivity check index). Additionally, curcumin further illustrated advantageous action with regards to enhancement of lipid profile, that was inclusive of, high density lipoprotein (HDL) as well as cholesterol. in con- trast to that not significantly alterations in low density lipoprotein (LDL) ovulation in addition to triglycerides (TG), in patients that consumed curcumin in contrast to placebo. The TSA illustrated that in this particular meta-analysis in the context of by fasting glucose, fasting insulin, HOMA- insulin resistance (IR) as well as QUICK1 in addition to TC were conclusive. As compared to the TSA pointed that the actions of curcumin on HDL, LDL, as well as TG are not conclusive till now, hence greater randomized larger scaled trials for estimation of these results (Figure 1).

Of the inclusive studies, the curcumin dosage varied from $500 \mathrm{mg}-1500 \mathrm{mg}$ daily, with treatment duration ranging from $6 \mathrm{wks}-$ $12 w k s$. The diagnosis of each patient was done with the utilization of Rotterdham criteria as well as evaluation of these patients was conducted in Iran. Of these, a study by Sohaei., et al. [6] gave the amount in addition to describe the manufacturing of curcumin with $95 \%$ of standardized turmeric powder. Despite, one study occurred in an infertility center however neither study considered to include studies that detailed the actions of curcumin in case of ovulation in addition to reproduction.

The actions of curcumin on glycemic regulation along with lipid metabolism are complicated, implicating numerous modes. i) Curcumin results in insulin modulated glucose uptake by the phospha- 


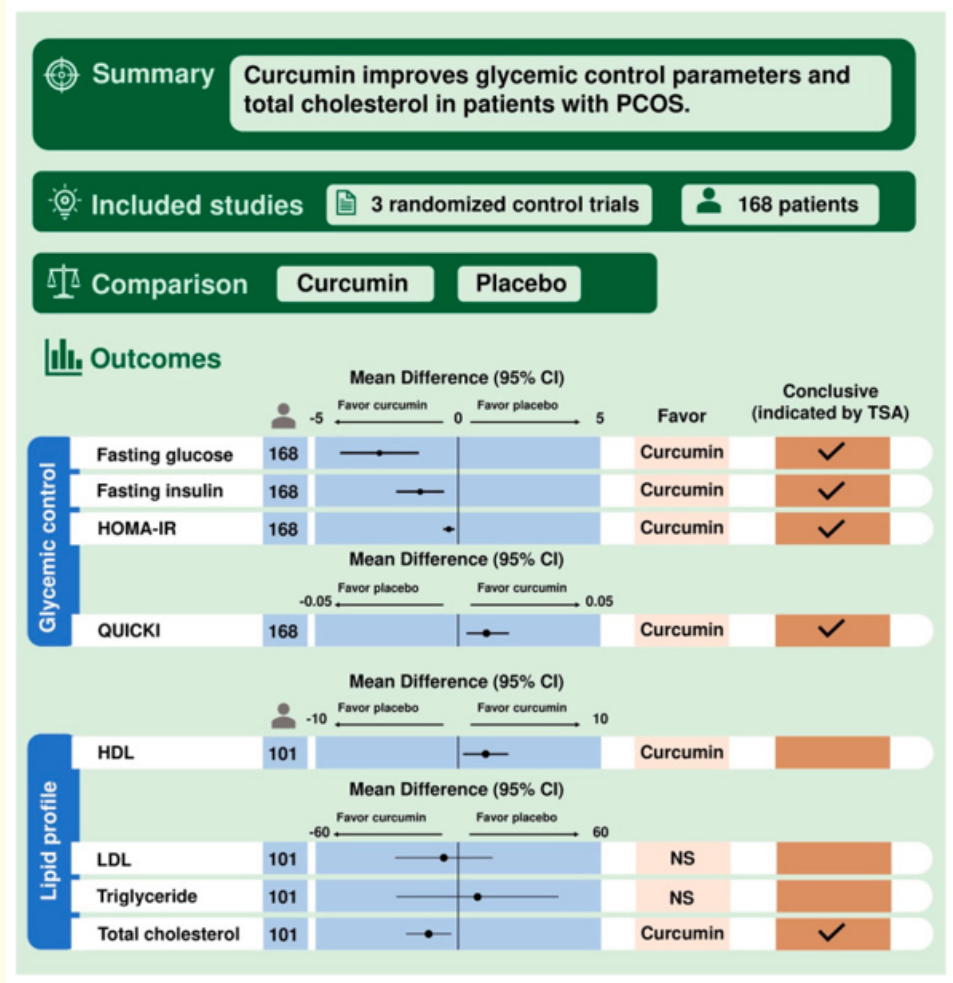

Figure 1: Courtesy ref no-5-Visual summary abstract.

CI: Confidence Interval, HDL: High-Density Lipoprotein, HOMA-IR: Homeostasis Model Assessment of Insulin Resistance, LDL: Low-Density Lipoprotein, NS: non-significant, PCOS: Polycystic Ovary Syndrome, QUICKI: Quantitative Insulin Sensitivity Check Index, TSA: Trial Sequential Analysis.

tidyl inositide 3 -kinase (PI3K)/protein kinase B (AKT) signaling pathway that in turn results in upregulation of the translocation of the glucose transporter 4 (GLUT4) to the membrane of adipocytes along with skeletal muscle that results in escalation of glucose uptake [7]. ii) Furthermore, curcumin causes stimulation of, 5' adenosine mono phosphate (AMP) -activated protein kinase (AMPK) that besides. iii) in repression gluconeogenesis in hepatocytes through hampering of glucose -6- phosphatase along with phospho enol pyruvate carboxy kinase (PEPCK) [8], causes enhancement of GLUT4 translocation as well as glucose uptake in adipocytes [9] (Figure 2). iv) Furthermore, curcumin enhancement of glucose homeostasis by stimulation of glucose transporter 2 in addition to v) glucokinases in liver by escalation of the transcription of Peroxisome Proliferator adenine. Activated Receptor gamma (PPAR $\gamma$ ) [10]. Jamilian., et al. [11], illustrated significant up regulation of PPAR $\gamma$ subsequent, to the intake of curcumin for12wks [11]. As far as the lipid metabolism is concerned up regulation of LDL Receptors, besides hampering the generation of triglycerides (TG), as well as cholesterol in hepatocytes is caused by curcumin [12]. Furthermore, curcumin facilitates cholesterol catabolism as well as faecal excretion of bile acids [13].

The anti-inflammatory characteristics of curcumin might play a part in glucose as well as lipid metabolism, besides might ameliorate hyperandrogenism. Proinflammatory cytokine, tumor necrosis factor alpha (TNF $\alpha)$ has been observed to be significantly greater in patients, with PCOS [14]. TNF $\alpha$ is understood to result in stimulation of serine phosphorylation of insulin receptor substrate (IRS1) that results in insulin resistance (IR) [15]. Furthermore, serine phosphorylation of cytochrome P450c17 causes escalation 


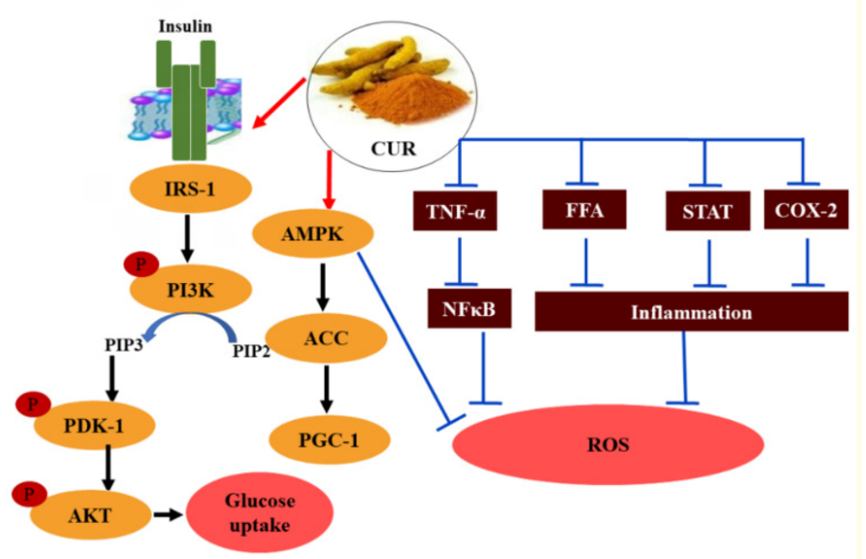

Figure 2: Courtesy ref no-26-Mechanisms of the potential antidiabetic effect of CUR.

IRS-1: Insulin Receptor Substrate-1, PI3K: Phosphatidyl Inositol-3-Kinase, PIP2: Phosphatidylinositol 4,5-Bisphosphate, PIP3: Phosphatidylinositol 3,4,5-Trisphosphate, PDKI:

Phosphoinositide-Dependent Protein Kinase 1, Akt: Protein

Kinase B, AMPK: Adenosine Monophosphate-Activated

Protein Kinase, ACC: Acetyl COA Carboxylase, PGC-1:

Peroxisome Proliferator-Activated Receptor-Gamma

Coactivator, TNF- $\alpha$ : Tumour Necrosis Factor-Alpha, NF- $\kappa$ B: Nuclear

Factor-Kappa B, FFA: Free Fatty Acids, STAT: Signal Transducer And Activator of Transcription, COX-2: Cyclooxygenase-2, ROS: Reactive Oxygen Species.

of the action of 17, 20lyase, thus facilitation of thecal generation of androgens [16]. Hampering of PI3K/Akt/ mTOR signaling pathway that causes break down of nuclear factor kappa light chain -enhancer of activated $\mathrm{B}$ cells (NFKB) in addition to down regulation of TNF $\alpha$ along with other proinflammatory cytokines $[17,18]$. Significant reduction of plasma amounts of $\mathrm{TNF} \alpha$ along with interleukin-6 (IL-6) has got illustrated by curcumin) [19] with subsequently causing enhancement of insulin sensitivity along with reduction of obesity stimulated insulin resistance [20]. Additionally, in patients with PCOS there is existence of Oxidative stress (OS) [21]. More recently, a study illustrated the advantageous action of curcumin on upregulation of gene expression of PPAR-gamma coactivator 1 alpha (PGC-1 $\alpha$ ) that in turn results in $g$ escalation of the action of glutathione peroxidase, thus resulting in reduction of Oxidative stress [22].
Although curcumin might be advantageous in relief from hyper androgenism, this particular study it was not demonstrated, in view of meta-analysis that causes assessment as well as documented the impact of curcumin on thecal generation of androgens had not been conducted in view of restricted studies. This study by Heshmati., et al. The plasma amounts of DHEA were significantly decreased in patients where consumption of curcumin was done as compared to those with intake of placebo for12 wks [23]. The cause of this observation might be secondary to the downregulation of cytochrome P450c17 action. It has been illustrated that curcumin causes reduction of the cytochrome P450c17 action that is in a dosage-based way in vitro [24]. utilization of an animal model cytochrome P450c17 in the ovaries of the patients in receipt of curcumin treatment in mice the reduction was to the akin extent as in the wild type ones [25]. Thus, together in addition to antiinflammatory characteristics of curcumin these observations validate the posit of reduction of cytochrome P450c17 action, as well as hence reduction of the generation of DHEA. Thus, the study by Heshmati J., et al. [23], validate the posit that curcumin might be resulting in reduction of androgens with its action of androgens of the cytochrome P450c17 action, hence in addition to probiotics might become an innovative therapy for PCOS women. More randomized, double blind placebo controlled Clinical trials are warranted by clinicians dealing with PCOS with regards to therapeutic role of curcumin.

\section{Bibliography}

1. Hong X., et al. "Association between Polycystic ovary syndrome and vaginal microbiome: a case-controlled study". Clinical Endocrinology 93 (2020b): 52-60.

2. Jin P and Xie Y. "Treatment strategies for women with Polycystic ovary syndrome". Gynecological Endocrinology 34 (2018): 272-277.

3. Kulvinder Kochar Kaur., et al. "How do we Manage Obese PCOS Infertile Patients”. EC Gynaecology 5 (5 (2017): 197-203.

4. Kulvinder Kochar Kaur., et al. "Initiation of a New Frontier in Obstetrics and Gynaecolgy - The Role of Vaginal Microbiota Dysbiosis in Case of Gynaecological Diseases and the Potential Treatment with Antibiotics, Probiotics and Vaginal Microbiota transplantation - How Practical Will it be - A Systematic Review". Acta Scientific Microbiology 4.9 (2021): 1-13.

5. Chien YJ., et al. "Effects of curcumin on glycaemic control and lipid profile in Polycystic ovary syndrome: systematic review with meta-analysis and trial sequential analysis". Nutrients 13 (2021): 684 . 
6. Sohaei S., et al. "The effects of curcumin supplementation on glycaemic status, lipid profile and hs-CRP levels in overweight/ obese women with Polycystic ovary syndrome: a randomized, double blind placebo controlled Clinical trial". Complementary Therapies in Medicine 47 (2019): 102201.

7. Den Hartogh DJ., et al. "Antidiabetic properties of curcumin I: evidence from in vitro studies". Nutrients 12 (2020): 118.

8. SrivastavaRA., et al. "AMP-activated protein kinase: an emerging drug target to regulate imbalances in carbohydrate and lipid metabolism to treat cardiometabolic diseases". Journal of Lipid Research 53 (2012): 2490-2514.

9. Yagamuchi S., et al. "Activators of AMP-activated protein kinase enhance GLUT4 translocation and its glucose transport activity in 3T3-L1 adipocytes". American Journal of Physiology-Endocrinology and Metabolism 289 (2005): E643-E649.

10. JacobA., et al. "Mechanisms of the anti-inflammatory Effects of curcumin: PPAR gamma activation". PPAR Research 2007 (2007): 89369.

11. JamilianM., et al. "Effects of curcumin on body weight, glycaemic control and serum lipids in in women within Polycystic ovary syndrome: a randomized double-blind placebo controlled Clinical trial". Clinical Nutrition ESPEN 36 (2020):128133.

12. Kim M and Kim Y. "Hypocholesterolemic Effects of curcumin via upregulation of cholesterol 7 ahydroxylase in rats fed a high fat diet". Research and Practice 4 (2010): 191-195.

13. Rao DS., et al. "Effects of curcumin on serum and liver cholesterol levels in the rat". Journal of Nutrition 100 (1970): 13071315.

14. Gao L., et al. "High serum tumor necrosis factor alpha i levels in women with Polycystic ovary syndrome: a Meta-Analysis". PLoS One 11 (2016): e0164021.

15. Rui L., et al. "Insulin/IGF1- and TNF $\alpha$ stimulate serine phosphorylation of IRS1 at inhibitory Ser 307 via distinct pathways". Journal of Clinical Investigation 107 (2001): 181-189.

16. Miller WK and Tee MK. "The post-translational regulation of 17,20 lyase activity". Molecular and Cellular Endocrinology 408 (2015): 90-106.

17. JobinC., et al. "Curcumin blocks cytokine mediated nuclear factor kappa B activation and proinflammatory gene expression by inhibiting inhibitory factor I kappa B kinase activity". Journal of Immunology 63 (1999): 3474-3483.
18. Zhang C., et al. "Curcumin induces apoptosis and inhibits angiogenesis in murine malignant mesothelioma”. International Journal of Oncology 53 (2018): 2531-2541.

19. Derosa G., et al. "Effects of curcumin on Circulating interleukin-6 Concentrations: a systematic review and, meta-analysis of randomized controlled trials". Pharmacological Research 107 (2016): 234-242.

20. Akash MSH., et al. "Tumor necrosis factor alpha: role in development of insulin resistance and pathogenesis of type2 Diabetes mellitus". Journal of Cellular Biochemistry 119 (2018): 105-110.

21. Sabancu T., et al. "Oxidative stress in Polycystic ovary syndrome and its contribution to the risk of cardiovascular disease". Clinical Biochemistry 34 (2001): 407-413.

22. Heshmati J., et al. "The effects of curcumin supplementation on Oxidative stress, Sirtuin 1 and Peroxisome Proliferator adenine Activated Receptor gamma coactivator 1 alpha gene expression in Polycystic ovary syndrome (PCOS) patients: a randomized placebo controlled Clinical trial". Diabetes and Metabolic Syndrome 14 (2020): 77-82.

23. Heshmati J., et al. "Effects of curcumin supplementation on blood glucose, insulin resistance and androgens in patients with Polycystic ovary syndrome: a randomized, double blind placebo controlled Clinical trial". Phytomedicine 80 (2020): 153395.

24. Rofdriquez-Castano., et al. "Bioactivity of curcumin on cytochrome P450c17 enzymes of the steroidogenic pathway". International Journal of Molecular Sciences 20 (2019): 105-4606.

25. Tiwari-Pandey R and Ram-Sairam. "Modulation of ovarian structure and abdominal obesity in curcumin and flutamidetreated aging FSH-R haploinsufficiency mice". Reproductive Sciences 16 (2009): 539-550.

26. Balbaa M., et al. "Therapeutic screening of herbal remedies for the management of Diabetes". Molecules 26 (2021): 6836.

\section{Assets from publication with us}

- Prompt Acknowledgement after receiving the article

- Thorough Double blinded peer review

- Rapid Publication

- Issue of Publication Certificate

- High visibility of your Published work

Website: www.actascientific.com/

Submit Article: www.actascientific.com/submission.php

Email us: editor@actascientific.com

Contact us: +919182824667 